\title{
Quantum entanglement of decohered two-mode squeezed states in absorbing and amplifying environment
}

\author{
Phoenix S. Y. Poon and C. K. Law \\ Department of Physics and Institute of Theoretical Physics, \\ The Chinese University of Hong Kong, Shatin, Hong Kong SAR, China
}

(Dated: November 5, 2018)

\begin{abstract}
We investigate the properties of quantum entanglement of two-mode squeezed states interacting with linear baths with general gain and loss parameters. By explicitly solving for $\rho$ from the master equation, we determine analytical expressions of eigenvalues and eigenvectors of $\rho^{T_{A}}$ (the partial transposition of density matrix $\rho$ ). In Fock space, $\rho^{T_{A}}$ is shown to maintain a block diagonal structure as the system evolves. In addition, we discover that the decoherence induced by the baths would break the degeneracy of $\rho^{T_{A}}$, and leads to a novel set of eigenvectors for the construction of entanglement witness operators. Such eigenvectors are shown to be time-independent, which is a signature of robust entanglement of two-mode squeezed states in the presence of noise.

PACS numbers: 03.67.Mn, 03.65.Yz, 42.50.Dv, 42.50.Lc
\end{abstract}

\section{INTRODUCTION}

Optical two-mode squeezed vacuum (TMSV) has been a major source of continuous-variable entanglement for quantum communication [1]. In recent years, intriguing applications such as quantum teleportation [2, 3, 4, 4, 5, 6] and quantum dense coding [7, 8] have been demonstrated experimentally with TMSV. Theoretically, it is also known that TMSV maximizes the EPR correlation when a fixed amount of entanglement is given [9]. In order to exploit fully the non-classical properties of such entangled light fields, it is important to understand decoherence effects as they propagate through noisy environments [10, 11, 12, 13, 14, 15, 16, 17, 18, 19]. This belongs to a more subtle topic involving the characterization and quantification of mixed state entanglement in general.

For bipartite systems, Peres and Horodecki have developed a powerful criterion of entanglement, which is known as the PPT (positive partial transposition) criterion [20, 21, 22]. If the partial transposition of a density matrix (denoted by $\rho^{T_{A}}$ ) has one or more negative eigenvalues, then the state is an entangled state. Physically, the partial transposition for separable states can be considered as a time-reversal operation, and one can construct a variety of uncertainty relations serving as indicators of entanglement [23, 24]. For two-mode Gaussian states such as TMSV, PPT provides a necessary and sufficient condition of separability 25, 26].

The dynamics of disentanglement of TMSV in various noisy situations has been addressed by several authors recently [10, 11, 12, 13, 14, 15, 16, 17, 18, 19]. The fact that an amplitude damped TMSV remains Gaussian enables an elegant description of entanglement based on the properties of covariance matrix associated with the density operators 27]. In particular, from the timedependent solution of Wigner function [15, 16, 17] or the corresponding characteristic function [19], one can quantify the degradation of entanglement by calculating the negativity [28] and relative entropy [29]. It is now known that for an initial TMSV at a non-zero thermal bath, quantum entanglement vanishes completely in a finite time [27].

However, we notice that there are much less investigations addressing the structure of $\rho^{T_{A}}$ directly, and yet $\rho^{T_{A}}$ is what the PPT criterion originally based upon. Since $\rho^{T_{A}}$ could manifest differently in various basis, the study of $\rho^{T_{A}}$ in Fock space, for example, could reveal entanglement properties not easily found by the Wigner function method [30]. An example we notice is the construction of entanglement witness operators via the projectors formed by the eigenvectors of $\rho^{T_{A}}$ with negative eigenvalues [31]. Such entanglement witness operators, which correspond a variety of observables for the detection of entanglement, are determined by $\rho^{T_{A}}$.

The main purpose of this paper to indicate some key features of decohered entanglement as revealed by eigenvalues and eigenvectors of $\rho^{T_{A}}$. Our analysis will concentrate on the structures of eigenvectors in Fock space, which is also where interesting non-local correlations of continuous-variable systems can be observed 32]. For a TMSV under the influence of amplitude damping (or gaining in an amplifier), we solve for the time evolution of $\rho$ and determine the exact eigenvectors and eigenvalues of $\rho^{T_{A}}$ analytically. These eigenvectors are shown to have a strong correlation in photon numbers, and hence $\rho^{T_{A}}$ is a block diagonal matrix in Fock space. Therefore witness operators associated with each block involve only a finite number of Fock vectors, which implies that the detection of entanglement can only require a small portion of the Hilbert space. This is in contrast to entanglement detection based on uncertainty relations in which the entire Hilbert space is usually involved [23, 24, 25, 26]. In this sense the eigenvectors of $\rho^{T_{A}}$ access the entanglement signatures 'locally', which is a complement to 'global' characterization (of Gaussian states) using covariance matrices. As we shall see below, as long as the initial state is a TMSV, the corresponding eigenvectors do not change with time, indicating that the entanglement carried by TMSV is robust against amplitude damping. 


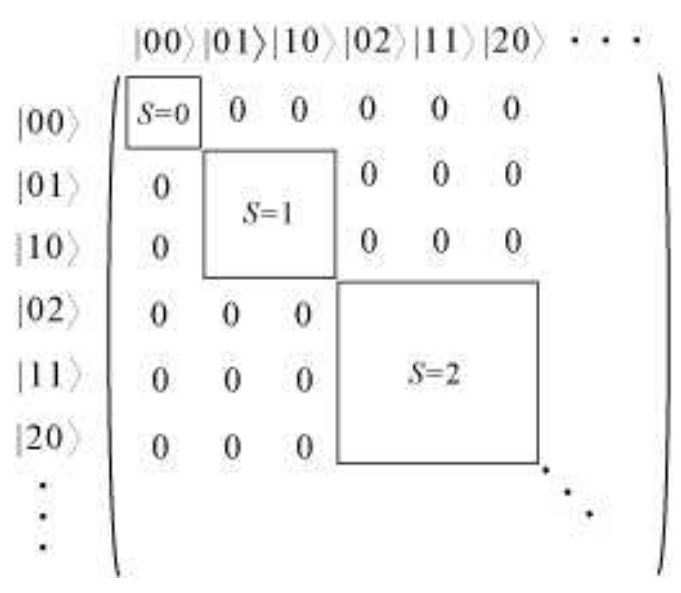

FIG. 1: The sub-matrix structure of $\rho^{T_{A}}$, with initial TMSV, in the Fock basis.

\section{MASTER EQUATION AND SOLUTION}

To begin with, we consider the time evolution of an initial TMSV, each coupled with a separate phaseinsensitive linear bath. In terms of the annihilation operators $a$ and $b$ of the two modes, the master equation governing the dynamical process is [33]:

$$
\begin{aligned}
\dot{\rho} & =G\left(2 a^{\dagger} \rho a-a a^{\dagger} \rho-\rho a a^{\dagger}+2 b^{\dagger} \rho b-b b^{\dagger} \rho-\rho b b^{\dagger}\right) \\
& +L\left(2 a \rho a^{\dagger}-a^{\dagger} a \rho-\rho a^{\dagger} a+2 b \rho b^{\dagger}-b^{\dagger} b \rho-\rho b^{\dagger} b\right)
\end{aligned}
$$

where $G$ and $L$ are the gain and loss parameters respectively, both having a dimension of time ${ }^{-1}$. Depending on the values of $G$ and $L$, the master equation describes amplifying or damping effects due to the coupling with the baths. For dissipation in thermal baths, each of temperature $T$, we have the parameters $G=\frac{\gamma}{2} n_{t h}$ and $L=\frac{\gamma}{2}\left(n_{t h}+1\right)$, where $n_{t h}=\frac{1}{\exp (h \omega / k T)-1}$ is the average number of photons in each of the modes (with frequency $\omega)$ at thermal equilibrium, and $\gamma / 2$ is the decay rate of the mode amplitudes. In this paper we focus on the initial TMSV with the squeezing parameter $r>0$ :

$$
|\psi(0)\rangle=\exp \left[r\left(a^{\dagger} b^{\dagger}-a b\right)\right]|00\rangle=\sqrt{1-\lambda^{2}} \sum_{n=0}^{\infty} \lambda^{n}|n n\rangle,(2)
$$

where $\lambda \equiv \tanh r$ and $|00\rangle$ is the two-mode vacuum state.

\section{A. Block structures of $\rho^{T_{A}}$ in Fock space}

To investigate the entanglement properties of the density matrix $\rho$, we study its partial transposition $\rho^{T_{A}}$. The $\rho^{T_{A}}$ is of infinite dimension, however, by examining the master equation in the Fock basis, block structures of $\rho^{T_{A}}$ can be identified. Let us denote the matrix elements of $\rho^{T_{A}}$ by

$$
\rho_{n, m, p, q}^{T_{A}}=\left\langle n m\left|\rho^{T_{A}}\right| p q\right\rangle=\langle p m|\rho| n q\rangle,
$$

which is governed by the following differential equation:

$$
\begin{aligned}
\dot{\rho}_{n, m, p, q}^{T_{A}}=G & {\left[2 \sqrt{n p} \rho^{T_{A}}{ }_{n-1, m, p-1, q}\right.} \\
& +2 \sqrt{m q} \rho^{T_{A}}{ }_{n, m-1, p, q-1} \\
& \left.-(n+m+p+q+4) \rho^{T_{A}}{ }_{n, m, p, q}\right] \\
+ & L\left[2 \sqrt{(n+1)(p+1)} \rho^{T_{A}}{ }_{n+1, m, p+1, q}\right. \\
& +2 \sqrt{(m+1)(q+1)} \rho^{T_{A}}{ }_{n, m+1, p, q+1} \\
& \left.-(n+m+p+q) \rho^{T_{A}}{ }_{n, m, p, q}\right] .
\end{aligned}
$$

and

$$
\rho_{n, m, p, q}^{T_{A}}(t=0)=\delta_{p m} \delta_{n q}\left(1-\lambda^{2}\right) \lambda^{m+n} .
$$

corresponds to the initial condition (2).

It can be seen from Eq. (4) that each element $\rho^{T_{A}}{ }_{n, m, p, q}(t)$ is coupled with elements $\rho^{T_{A}}{ }_{n+l, m+k, p+l, q+k}(0)$ only, for integers $l$ and $k$. Therefore $\rho^{T_{A}}{ }_{n+l, m+k, m+l, n+k}(t)$ are the only non-zero elements at any time $t>0$ because of the initial condition. By noting that the sum of the first two indices equal to that of the last two, we can group all non-zero elements $\rho^{T_{A}}{ }_{n+l, m+k, m+l, n+k}(t)$ into sub-matrices $\mathcal{M}_{S}$ according to the sum index $S=n+l+m+k$, i.e., $\rho^{T_{A}}{ }_{n+l, m+k, m+l, n+k}(t)$ is contained in $\mathcal{M}_{n+l+m+k}$. We can therefore express $\rho^{T_{A}}$ in a direct sum of $\mathcal{M}_{S}$ as follows:

$$
\rho^{T_{A}}(t)=\bigoplus_{S=0}^{\infty} \mathcal{M}_{S}(t)
$$

where the sub-matrix $\mathcal{M}_{S}$ has a dimension of $S+1$, since elements in $\mathcal{M}_{S}$ have its first two indices as $\{0, S\},\{1, S-$ $1\}, \ldots,\{S, 0\}$. Fig. 1 shows the sub-matrix structure of $\rho^{T_{A}}$. Note that characteristic sum $S$ is equal to the total number of photons that the two modes contain. From Eq. (4) we observe that probabilistic flow occurs between elements in neighboring sub-matrices, with emission or absorption of one photon in one of the modes at one time.

The time evolution of a typical sub-matrix of $\rho^{T_{A}}$ is illustrated schematically in Fig. 2, which will be discussed in detail in the later part of the paper. Initially, only opposite-diagonal elements are present, having the magnitude as $\lambda^{S}$. As time increases, element flows from neighboring sub-matrices, and disentanglement of the sub-matrices occurs at a critical time $t=$ $t_{c}$ (Section ஹIA). In the case of thermal bath, $\rho^{T_{A}}$ evolves into a diagonal form in the long time limit, settling as the thermal equilibrium state $\rho_{n, m, p, q}^{T_{A}}=$ $\delta_{n p} \delta_{m q} \frac{1}{\left(n_{t h}+1\right)^{2}}\left(\frac{n_{t h}}{n_{t h}+1}\right)^{n+m}$.

\section{B. Analytic solution of $\rho$ in position space}

To analyze the properties of $\rho^{T_{A}}$, it is more convenient to first determine $\rho$ in position space and then 


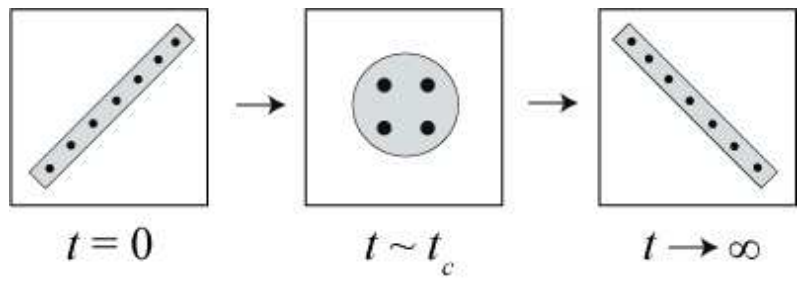

FIG. 2: (Color online) Schematic diagram showing the evolution of the distribution of elements in a sub-matrix of $\rho^{T_{A}}$, assuming the baths are thermal baths. The $t_{c}$ is the critical time for disentanglement.

make the transformation to Fock space. The position space method of finding $\rho$ was previously employed in Ref. [11, 34] in studying entanglement in various oscillator systems. In this subsection, we present an explicit solution of master equation (1) with an initial TMSV. We remark that our method is different from that given in [11], as the latter involves a Fourier transform of the density matrix, i.e., the momentum space. Here we solve the density matrix entirely in position space (Appendix A). This turns out to be more convenient for the real symmetric Gaussian states considered here, since fewer differential equations are involved. In addition, the resultant solution is more transparent for further analysis of eigenvectors in the next section.

Let us denote the 'position' operators as $x=\frac{1}{\sqrt{2}}\left(a+a^{\dagger}\right)$ and $y=\frac{1}{\sqrt{2}}\left(b+b^{\dagger}\right)$, and define

$$
\rho\left(x_{1}, y_{1} ; x_{2}, y_{2} ; t\right) \equiv\left\langle x_{1}, y_{1}|\rho(t)| x_{2}, y_{2}\right\rangle,
$$

then the master equation (1) becomes,

$$
\begin{aligned}
\dot{\rho}= & -\frac{1}{2}\left[L \left(x_{1}^{2}+x_{2}^{2}-2 x_{1} x_{2}+y_{1}^{2}+y_{2}^{2}-2 y_{1} y_{2}-4\right.\right. \\
& -\partial_{x_{1}}^{2}-\partial_{x_{2}}^{2}-\partial_{y_{1}}^{2}-\partial_{y_{2}}^{2}-2 \partial_{x_{1}} \partial_{x_{2}}-2 \partial_{y_{1}} \partial_{y_{2}} \\
& \left.-2 x_{1} \partial_{x_{2}}-2 x_{2} \partial_{x_{1}}-2 y_{1} \partial_{y_{2}}-2 y_{2} \partial_{y_{1}}\right) \\
& +G\left(x_{1}^{2}+x_{2}^{2}-2 x_{1} x_{2}+y_{1}^{2}+y_{2}^{2}-2 y_{1} y_{2}+4\right. \\
& -\partial_{x_{1}}^{2}-\partial_{x_{2}}^{2}-\partial_{y_{1}}^{2}-\partial_{y_{2}}^{2}-2 \partial_{x_{1}} \partial_{x_{2}}-2 \partial_{y_{1}} \partial_{y_{2}} \\
& \left.\left.+2 x_{1} \partial_{x_{2}}+2 x_{2} \partial_{x_{1}}+2 y_{1} \partial_{y_{2}}+2 y_{2} \partial_{y_{1}}\right)\right] \rho .
\end{aligned}
$$

For an initial state $(2), \rho\left(x_{1}, y_{1} ; x_{2}, y_{2} ; t\right)$ takes a Gaussian form at any time $t$,

$$
\begin{aligned}
\rho\left(x_{1}, y_{1} ; x_{2}, y_{2} ; t\right)= & \Xi(t) \exp \left[-A(t)\left(x_{1}^{2}+x_{2}^{2}+y_{1}^{2}+y_{2}^{2}\right)\right. \\
& +B(t)\left(x_{1} y_{1}+x_{2} y_{2}\right) \\
& +C(t)\left(x_{1} x_{2}+y_{1} y_{2}\right) \\
& \left.+D(t)\left(x_{1} y_{2}+x_{2} y_{1}\right)\right]
\end{aligned}
$$

where $A(t), B(t), C(t)$ and $D(t)$ are real time-dependent coefficients, and the normalization factor is:

$$
\Xi(t)=\frac{1}{\pi} \sqrt{[2 A(t)-C(t)]^{2}-[B(t)+D(t)]^{2}} .
$$

By substituting Eq. (9) into the master equation, the coefficients are found to obey a set of coupled equations that can be solved analytically (Appendix A). For the TMSV considered here, we have,

$$
\begin{aligned}
A(t) & =\frac{1}{4}\left[2 A_{0} \eta+\frac{G+L}{G-L}(\eta-1)+\frac{\left\langle x^{2}\right\rangle_{t}}{2\left(\left\langle x^{2}\right\rangle_{t}^{2}-\langle x y\rangle_{t}^{2}\right)}\right], \\
B(t) & =\frac{1}{2}\left[B_{0} \eta+\frac{\langle x y\rangle_{t}}{2\left(\left\langle x^{2}\right\rangle_{t}^{2}-\langle x y\rangle_{t}^{2}\right)}\right], \\
C(t) & =\frac{1}{2}\left[2 A_{0} \eta+\frac{G+L}{G-L}(\eta-1)-\frac{\left\langle x^{2}\right\rangle_{t}}{2\left(\left\langle x^{2}\right\rangle_{t}^{2}-\langle x y\rangle_{t}^{2}\right)}\right], \\
D(t) & =\frac{1}{2}\left[-B_{0} \eta+\frac{\langle x y\rangle_{t}}{2\left(\left\langle x^{2}\right\rangle_{t}^{2}-\langle x y\rangle_{t}^{2}\right)}\right] .
\end{aligned}
$$

Here $A_{0}=\frac{1}{2} \cosh 2 r, B_{0}=\sinh 2 r$ and $\eta(t)=\exp [2(G-$ $L) t]$ are defined, and the expectation values are given by,

$$
\begin{aligned}
\left\langle x^{2}\right\rangle_{t} & =A_{0} \eta+\frac{G+L}{2(G-L)}(\eta-1), \\
\langle x y\rangle_{t} & =\frac{B_{0}}{2} \eta .
\end{aligned}
$$

\section{PROPERTIES OF $\rho^{T_{A}}$}

According to PPT criterion, the appearance of negative eigenvalues of $\rho^{T_{A}}$ is a signature of entanglement. In this section, we solve the eigenvectors and eigenvalues of $\rho^{T_{A}}$ as the system evolves. Then we discuss how decoherence affects the entanglement properties of $\rho^{T_{A}}$. The eigenvalues and eigenvectors of $\rho^{T_{A}}$ are defined by:

$$
\begin{aligned}
& \iint \rho^{T_{A}}\left(x_{1}, y_{1} ; x_{2}, y_{2} ; t\right) \varphi_{n, m}\left(x_{2}, y_{2} ; t\right) d x_{2} d y_{2} \\
& =\xi_{n, m}(t) \varphi_{n, m}\left(x_{1}, y_{1} ; t\right) .
\end{aligned}
$$

Our main technique of solving the eigen-problem is the use of Mehler formula which expands a double Gaussian function into a series of orthogonal functions. After some calculations (see Appendix B), we obtain the expression of eigenvalues,

$$
\xi_{n, m}(t)=\Xi(t) \pi \frac{\left(\sqrt{\alpha_{1}}-\sqrt{\beta_{1}}\right)^{n}}{\left(\sqrt{\alpha_{1}}+\sqrt{\beta_{1}}\right)^{n+1}} \frac{\left(\sqrt{\alpha_{2}}-\sqrt{\beta_{2}}\right)^{m}}{\left(\sqrt{\alpha_{2}}+\sqrt{\beta_{2}}\right)^{m+1}}
$$

and the corresponding eigenvectors,

$$
\begin{aligned}
\varphi_{n, m}\left(x_{1}, y_{1}\right)= & \frac{1}{\sqrt{2^{n+m} n ! m ! \pi}} H_{n}\left(\frac{x_{1}-y_{1}}{\sqrt{2}}\right) H_{m}\left(\frac{x_{1}+y_{1}}{\sqrt{2}}\right) \\
& \times \exp \left[-\frac{1}{2}\left(x_{1}^{2}+y_{1}^{2}\right)\right]
\end{aligned}
$$

where $H_{n}$ are the Hermite polynomials. In writing Eq. (14), we have defined

$$
\begin{aligned}
& \alpha_{1}(t)=\frac{1}{4}[2 A(t)-B(t)+C(t)+D(t)], \\
& \beta_{1}(t)=\frac{1}{4}[2 A(t)+B(t)-C(t)+D(t)], \\
& \alpha_{2}(t)=\frac{1}{4}[2 A(t)+B(t)+C(t)-D(t)], \\
& \beta_{2}(t)=\frac{1}{4}[2 A(t)-B(t)-C(t)-D(t)]
\end{aligned}
$$


and the solution of $A, B, C$ and $D$ are given by Eq. (11). Note that in writing Eq. (15) from (B8), we have used the fact that for TMSV, $\alpha_{1} \beta_{1}=\alpha_{2} \beta_{2}=\frac{1}{16}$ for all time $t \geq 0$.

We now transform the eigenvectors from the position space to the Fock space. Note that $\rho^{T_{A}}$ is a basis dependent operation. The eigenvectors of $\rho^{T_{A}}$ defined in two different basis sets do not transform directly. An exception is the case when the two sets of basis vectors transform by a real unitary matrix 35], which is the case here. This allows us to write down the eigenvector $\left|\varphi_{n, m}\right\rangle$ in Fock space from Eq. (15):

$$
\left|\varphi_{n, m}\right\rangle=\left[\frac{1}{\sqrt{n !}}\left(\frac{a^{\dagger}-b^{\dagger}}{\sqrt{2}}\right)^{n}\right]\left[\frac{1}{\sqrt{m !}}\left(\frac{a^{\dagger}+b^{\dagger}}{\sqrt{2}}\right)^{m}\right]|00\rangle,
$$

which can be connected to the sub-matrices of $\rho^{T_{A}}$ (Fig. 1) via the photon number sum $S \equiv m+n$ to label the eigenket, so that

$$
\begin{aligned}
\left|\varphi_{n, S-n}\right\rangle= & \frac{1}{\sqrt{2^{S}} \sqrt{n !(S-n) !}} \\
& \times \sum_{j=0}^{S} \Gamma_{S, n, j} \sqrt{j !(S-j) !}|j, S-j\rangle .
\end{aligned}
$$

Here we have used the abbreviation

$$
\Gamma_{S, n, j} \equiv \sum_{k=0}^{\min (j, n)}(-1)^{n-k} C_{j-k}^{S-n} C_{k}^{n},
$$

with $C_{r}^{S} \equiv \frac{S !}{r !(S-r) !}$. In this way $\left|\varphi_{n, S-n}\right\rangle$ and $\xi_{n, S-n}$ $(n=0,1, \ldots, S)$ are the $n^{\text {th }}$ eigenvector and eigenvalue of the block with characteristic sum $S$.

\section{A. Evolution of negative eigenvalues}

By inspecting Eq. (14), we find that all the negative eigenvalues in each block share the same value at $t=0$ (Fig. 3). The same is true also for positive eigenvalues. However, such a strong degeneracy is broken by coupling with the baths. This is illustrated in Fig. 3 where the time-dependence of individual eigenvalues in various block indices $S$ is shown. Except at $t=0$ and at the critical time $t_{c}$, we see that the negative eigenvalues possessing different values.

It is important to observe that the eigenvalues are negative for odd $n$, when $\sqrt{\alpha_{1}}<\sqrt{\beta_{1}}$, or in other words, $B(t)>C(t)$. All eigenvalues turn zero at the same time, when we have $B(t)=C(t)$, except for the only eigenvalue with $n=0$ in each block. Such a critical time $t_{c}$ is given by,

$$
t_{c}=\frac{1}{2(L-G)} \log \left(\frac{G+L \lambda}{G(1+\lambda)}\right)
$$

which is always positive finite as long as $G \neq 0$. For the case $G \rightarrow 0$, we have $t_{c} \rightarrow \infty$. We remark that the
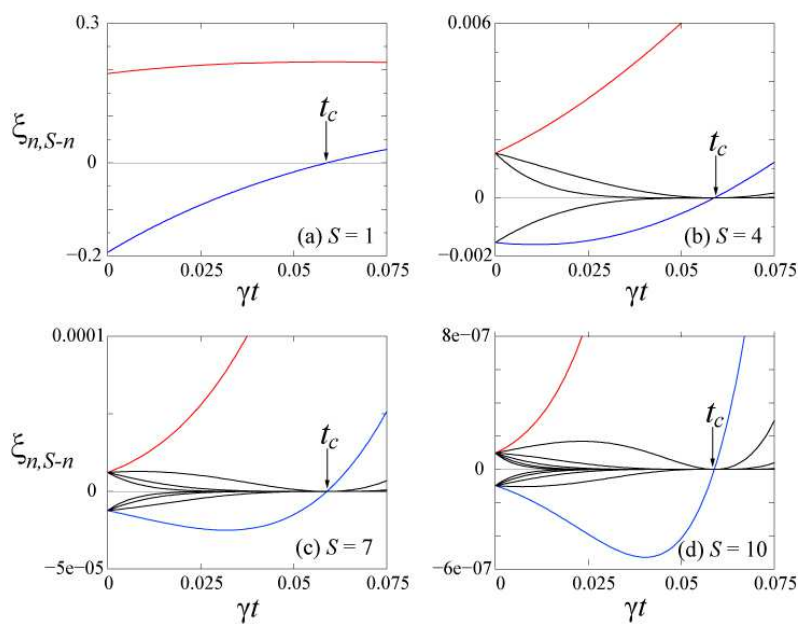

FIG. 3: (Color online) The eigenvalues of sub-matrices of $\rho^{T_{A}}$ of an amplifier system with $G=1.5 \gamma, L=0.5 \gamma$ and initial squeezing factor $r=\tanh ^{-1} 0.2$, with characteristic sum (a) $S=1$, (b) $S=4$, (c) $S=7$, and (d) $S=10$. Red line indicates the $n=0$ eigenvalue which does not turn zero at $t_{c}$, while blue line indicates the most negative eigenvalue with $n=1$.

disentanglement time $t_{c}$ was previous obtained in Ref. 26] for thermal baths, here we obtained a general expression (20) that applies to linear amplifiers as well. In particular, in the case when gain and loss parameters are equal, i.e., $G=L$, the critical time can be reduced to $t_{c}=\frac{1}{2 G} \frac{\lambda}{1+\lambda}>0$.

We point out that at the time of disentanglement $t=$ $t_{c}$, there is only one non-zero eigenvalue (with the index $n=0$ ) in each sub-matrix (Fig. 31). Therefore $\rho^{T_{A}}$ at the critical time is highly degenerate, and the corresponding symmetry property of $\rho^{T_{A}}\left(t=t_{c}\right)$ is indicated in the relation:

$$
\begin{aligned}
& \rho^{T_{A}}{ }_{j, S-j, j, S-j}=\rho^{T_{A}}{ }_{j, S-j, S-j, j} \\
& =\rho^{T_{A}}{ }_{S-j, j, j, S-j}=\rho^{T_{A}}{ }_{S-j, j, S-j, j} .
\end{aligned}
$$

This results in the symmetric distribution of elements as shown in Fig. 2 schematically.

As a further remark, it is interesting that negative eigenvalues may not necessarily be monotones over time. This can be seen by differentiating Eq. (14) and looking at the initial rate:

$$
\begin{aligned}
\left.\dot{\xi}_{n, S-n}\right|_{t=0}= & (-1)^{n} \tanh ^{S} r \operatorname{csch} r \operatorname{sech}^{3} r \\
\times & \{(G-L)(S-2 n) \\
& +(G+L)(S-2 n) \cosh 2 r \\
& -[L S+G(2+S)] \sinh 2 r\},
\end{aligned}
$$

which can result in a negative value for certain parameters, i.e., some eigenvalues of odd $n$ can become more negative over time (Fig. 3 $\mathrm{d}$ ). An exceptional case is when $G=0$, where we find that for odd $n$, the derivative at $t=0$ must be positive by inspecting Eq. (22). 


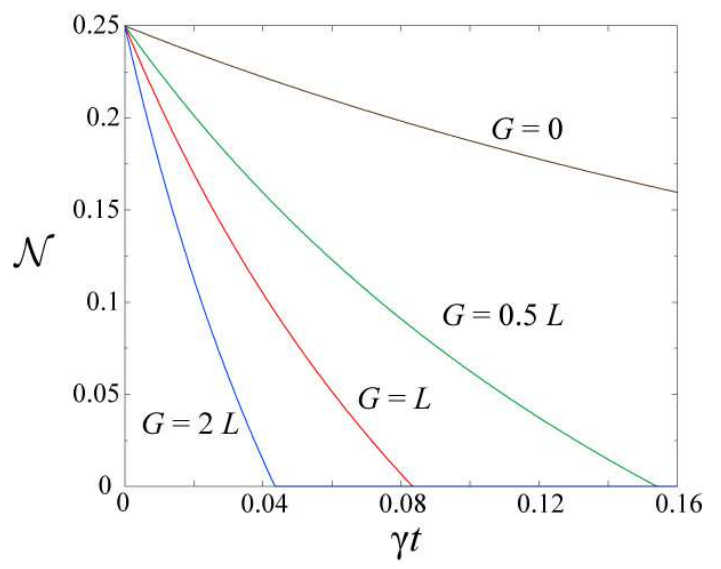

FIG. 4: (Color online) The negativity $\mathcal{N}$ of $\rho^{T_{A}}$ with different $G$, fixing the parameters $L=\gamma$ and initial squeezing factor $r=\tanh ^{-1} 0.2$.

\section{B. Negativity and sub-negativity}

Negativity $\mathcal{N}$ serves as a computable measure of entanglement defined by the trace norm of $\rho^{T_{A}}$ minus 1 divided by 2 [28]. For separable states, $\rho^{T_{A}}$ is still a density matrix with trace 1 and hence $\mathcal{N}=0$. However, for non-separable states with negative $\rho^{T_{A}}$, we have $\mathcal{N}>0$. Specifically, $\mathcal{N}$ equals the sum of the absolute value of negative eigenvalues of $\rho^{T_{A}}[28,36]$. For the system considered in this paper, $\mathcal{N}$ reads,

$$
\mathcal{N}=\frac{\pi \Xi(t)}{8}\left(\frac{1}{\sqrt{\alpha_{1} \beta_{2}}}-\frac{1}{\sqrt{\beta_{1} \beta_{2}}}\right) .
$$

Alternatively, $\mathcal{N}$ can be derived from the symplectic spectrum of the covariance matrix associated with the density operator [27]. In Fig. 4 we show the time evolution of negativity $\mathcal{N}$ for initial TMSV with different $G$ parameters. An example for the $G=0$ case is the zerotemperature bath dissipation scenario. Fixing $L$, we observe that a larger gain $G$ leads to a shorter $t_{c}$.

We can also calculate the negativity in a sub-matrix $\mathcal{M}_{S}$, which measures the contribution of entanglement from the corresponding block that builds up $\rho^{T_{A}}$. Specifically, the sub-matrix negativity $\mathcal{N}_{S}$ is defined the same way as negativity $\mathcal{N}$ but restricted to the sub-matrix of $\rho^{T_{A}}$ with the characteristic sum $S$. To our knowledge, such a sub-matrix negativity, which requires the calculations of individual eigenvalues, has not been discussed before. Explicitly, $\mathcal{N}_{S}$ takes the form:

$\mathcal{N}_{S}=-\pi \Xi(t) \sum_{n=0}^{P} \frac{\left(\sqrt{\alpha_{1}}-\sqrt{\beta_{1}}\right)^{2 n+1}}{\left(\sqrt{\alpha_{1}}+\sqrt{\beta_{1}}\right)^{2 n+2}} \frac{\left(\sqrt{\alpha_{2}}-\sqrt{\beta_{2}}\right)^{S-2 n-1}}{\left(\sqrt{\alpha_{2}}+\sqrt{\beta_{2}}\right)^{S-2 n}}$

where $P$ is defined as the integral part of $\frac{S-1}{2}$. In Fig. 5 the behavior of $\mathcal{N}_{S}$ of some blocks is illustrated. It is surprising that that for higher sub-matrices the corresponding negativity $\mathcal{N}_{S}$ may increase over time. Physically, the increase of $\mathcal{N}_{S}$ is due to the probability flow in
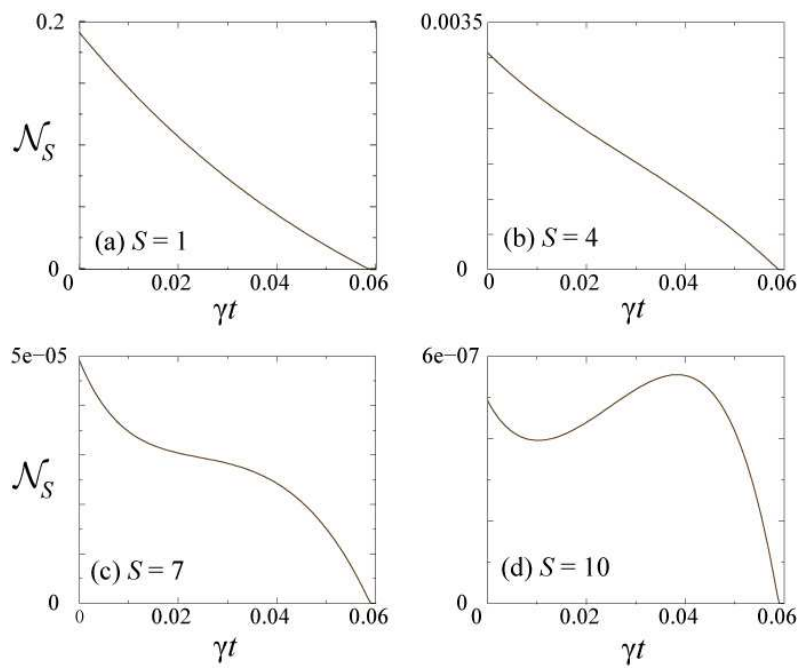

FIG. 5: The negativity of sub-matrices $\mathcal{N}_{S}$ of $\rho^{T_{A}}$, for an amplifier system with $G=1.5 \gamma, L=0.5 \gamma$ and initial squeezing factor $r=\tanh ^{-1} 0.2$, with characteristic sum (a) $S=1$, (b) $S=4,(\mathrm{c}) S=7$, and (d) $S=10$.

Fock space arising from damping or amplifying mechanisms. Since each sub-matrix of $\rho^{T_{A}}$ does not necessarily conserve probability (i.e., the trace of $\mathcal{M}_{S}$ is not a constant), it is possible that some blocks could have their negativity increasing with time.

However, the increase of $\mathcal{N}_{S}$ does not violate the fact that the overall negativity $\mathcal{N}$ of $\rho^{T_{A}}$ is an entanglement monotone that does not increase under LOCC (the master equation corresponds to local operations). Eq. (14) reveals that eigenvalues of higher blocks are of smaller order of magnitude. As we see in Fig. 5, although negativity of individual higher sub-matrices may increase over time, their contribution for negativity is smaller by several orders than the sub-matrices with lower $S$, and therefore the overall negativity is still monotonic decreasing.

\section{Robust structure of entanglement witness}

An entanglement witness operator $\mathcal{W}$ is designed for the detection of entanglement such that $\operatorname{Tr}(\rho \mathcal{W})<0$ for some non-separable states $\rho$, but $\operatorname{Tr}\left(\rho_{\text {sep }} \mathcal{W}\right) \geq 0$ for all separable states $\rho_{\text {sep }}$ 21]. For each eigenvector $|\phi\rangle$ of $\rho^{T_{A}}$ with a negative eigenvalue, one can construct an $\mathcal{W}$ by $\mathcal{W}=|\phi\rangle\left\langle\left.\phi\right|^{T_{A}}\right.$ 31] meeting the criteria above. In our system, we can construct a family of $\mathcal{W}$ from eigenvectors $\left|\varphi_{n, S-n}\right\rangle$ with odd $n$ accordingly, i.e., $\mathcal{W}_{S, n}=\left|\varphi_{n, S-n}\right\rangle\left\langle\left.\varphi_{n, S-n}\right|^{T_{A}}\right.$. From Eq. (18), the explicit form of $\mathcal{W}_{S, n}$ reads,

$$
\begin{aligned}
\mathcal{W}_{S, n}= & \sum_{j=0}^{S} \sum_{l=0}^{S}\left\{\frac{1}{2^{S} n !(S-n) !} \Gamma_{S, n, j} \Gamma_{S, n, l}\right. \\
& \times \sqrt{j !(S-j) ! l !(S-l) !}\}|l, S-j\rangle\langle j, S-l|,
\end{aligned}
$$




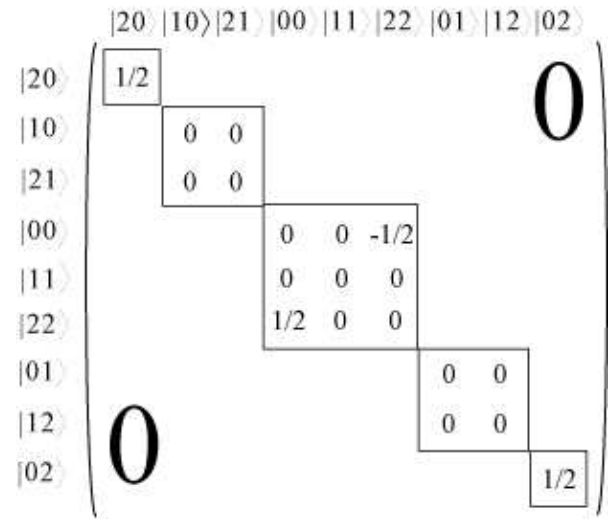

FIG. 6: Example of entanglement witness $\mathcal{W}_{2,1}$.

which shows that $\mathcal{W}_{S, n}$ operates in only a finite dimension in the Fock space. Note that $\operatorname{Tr}\left(\rho \mathcal{W}_{S, n}\right)=\xi_{n, S-n}$, and $\xi_{1, S-1}$ is the most negative eigenvalue in each submatrix, therefore $\mathcal{W}_{S, 1}$ provides the most significant entanglement detection among all witnesses constructed from vectors lying within the sub-matrix $\mathcal{M}_{S}$. Some examples for entanglement witnesses with $n=1$ are shown in Fig. 6 and Fig. 7 .

We observe that $\mathcal{W}$ also has a block diagonal structure in which non-zero elements are: $\left\langle j, S-l\left|\mathcal{W}_{S, n}\right| l, S-j\right\rangle$. This allows us to divide $\mathcal{W}$ into sub-blocks $\Lambda_{K}$, with each sub-block characterized by a difference $K \in[-S, S]$ :

$$
\mathcal{W}_{S, n}=\bigoplus_{K=-S}^{S} \Lambda_{K},
$$

where the element $\left\langle j, S-l\left|\mathcal{W}_{S, n}\right| l, S-j\right\rangle$ lies in the subblock with $K=S-j-l$. The explicit form of $\Lambda_{K}$ is

$$
\begin{aligned}
\Lambda_{K}= & \sum_{l=\max (0,-K)}^{\min (S, S-K)}\left\{\frac{1}{2^{S} n !(S-n) !} \Gamma_{S, n, S-K-l} \Gamma_{S, n, l}\right. \\
& \times \sqrt{(S-K-l) !(K+l) ! l !(S-l) !}\} \\
& |l, K+l\rangle\langle S-K-l, S-l|
\end{aligned}
$$

having a dimension of $S-|K|+1$. We remark that the simplest $2 \times 2$ entanglement witness $\mathcal{W}_{1,1}$ was constructed in [37] using a different approach. Here our general $\mathcal{W}_{S, n}$ applies to all $S$ and $n$.

Finally, let us emphasize the robustness feature of TMSV against decoherence. We have seen from Eq. (18) explicitly that the eigenvectors $\left|\varphi_{n, S-n}\right\rangle$ remain unchanged with time. The time-independent $\left|\varphi_{n, S-n}\right\rangle$ suggests that TMSV is robust against noise, in the sense that structure of entanglement witness $\mathcal{W}_{S, n}$ is preserved. The degradation of entanglement would only affect the eigenvalues. We stress that such a time-independent property of eigenvectors is specific to initial TMSV, and does not hold for arbitrary initial states in general. In Appendix B. we derive the eigenvectors of $\rho^{T_{A}}$ evolving from an initial two-mode symmetric Gaussian states with arbitrary

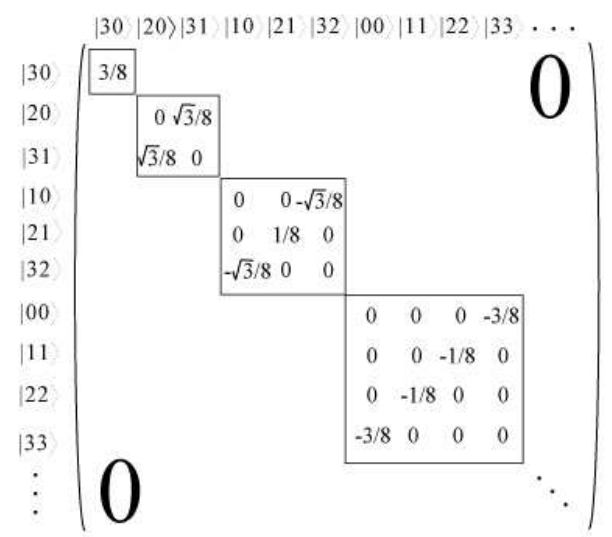

FIG. 7: Part of the entanglement witness $\mathcal{W}_{3,1}$, showing the blocks with characteristic difference from -3 to $0 . \mathcal{W}_{3,1}$ is symmetric about the top-right to bottom-left diagonal.

real coefficients $A_{0}, B_{0}, C_{0}$ and $D_{0}$. We find that the time dependence of the eigenvectors arises solely from the evolution of the factors $\alpha_{1} \beta_{1}$ and $\alpha_{2} \beta_{2}$. Because of the system-bath interactions, these factors are generally dependent on time. However, the state evolving from TMSV is an exception in which one can show that the corresponding $\alpha_{1} \beta_{1}$ and $\alpha_{2} \beta_{2}$ equal the constant $1 / 16$ at all times.

\section{CONCLUSION}

To summarize, by deriving an exact analytic solution for $\rho^{T_{A}}$ and examining its eigenvectors, we discover several important features about the loss of entanglement of a TMSV suffered from decoherence. Both amplitude damping and amplification effects have been included in our analysis. Throughout the decoherence process, the block diagonal structure of $\rho^{T_{A}}$ is shown to be maintained in Fock basis. As each block spans only a finite portion of the Fock space, the existence of negative eigenvalues in a block implies that entanglement information can 'survive' in the corresponding photon-number subspace. If quantum entanglement of the system is to be destroyed completely, then all the blocks have to be made positive. In other words, by simply mixing the system with another state involving finite Fock space would not destroy the entanglement. For the decoherence process considered in this paper, all the blocks turn positive at a critical time $t_{c}$, which agree with the previous analysis based on the covariance matrix. At $t<t_{c}$, we derived an explicit expression of the negativity as well as the negativity of sub-matrices in order to characterize the time-dependence of entanglement. Interestingly, the negativity of some sub-matrices could increase with time when $G$ is non-zero, although the effect is weak according to our calculations. The signature of entanglement in photon number subspace can be detected by the witness operators $\mathcal{W}_{S, n}$, and we have constructed $\mathcal{W}_{S, n}$ explicitly 
in this paper. These witness operators are also block diagonal, and most remarkably, they are time-independent even though the system is under the influence of noise. We interpret such a property as a kind of robust entanglement structure inherited in TMSV.

\section{Acknowledgments}

This work is supported by the Research Grants Council of the Hong Kong SAR, China (Project No. 401305).

\section{APPENDIX A: TIME EVOLUTION OF GENERAL REAL SYMMETRIC TWO-MODE GAUSSIAN DENSITY OPERATOR}

In this Appendix, we consider the time evolution of real symmetric two-mode Gaussian states, each coupling linearly with a separate bath with general gain and loss parameters. The density matrix is represented in position space, obeying the master equation as in Eq. (8). For the solution of the two-mode real symmetric Gaussian state presented in Eq. (9), a direct substitution leads to the following coupled differential equations:

$$
\begin{aligned}
\dot{A} & =(G-L) C+\frac{1}{2}(G+L)\left[1-(2 A-C)^{2}-(B+D)^{2}\right], \\
\dot{B} & =-2(G-L) D-2(G+L)(2 A-C)(B+D), \\
\dot{C} & =4(G-L) A+(G+L)\left[1+(2 A-C)^{2}+(B+D)^{2}\right], \\
\dot{D} & =-2(G-L) B-2(G+L)(2 A-C)(B+D) . \quad(\mathrm{A} 1)
\end{aligned}
$$

Without loss of generality, we consider the $G \neq L$ case. From Eqs. (A1), $B-D$ and $2 A+C$ have the simple solution,

$$
\begin{aligned}
B(t)-D(t) & =\left(B_{0}-D_{0}\right) \eta(t), \\
2 A(t)+C(t) & =\left(2 A_{0}+C_{0}\right) \eta(t)+\frac{G+L}{G-L}[\eta(t)-1],
\end{aligned}
$$

where $\eta(t) \equiv \exp [2(G-L) t]$ and the zero subscripts denote the values at $t=0$. The other two combinations, $B+D$ and $2 A-C$, can be found by noting that they are related to the second moments $\left\langle x^{2}\right\rangle$ and $\langle x y\rangle$ by:

$$
\begin{aligned}
2 A(t)-C(t) & =\frac{\left\langle x^{2}\right\rangle_{t}}{2\left[\left\langle x^{2}\right\rangle_{t}^{2}-\langle x y\rangle_{t}^{2}\right]}, \\
B(t)+D(t) & =\frac{\langle x y\rangle_{t}}{2\left[\left\langle x^{2}\right\rangle_{t}^{2}-\langle x y\rangle_{t}^{2}\right]} .
\end{aligned}
$$

with the subscript $t$ denoting the value at time $t$. Under the condition:

$$
\left\langle a^{2}\right\rangle_{t}=\left\langle b^{2}\right\rangle_{t}=\left\langle a^{\dagger} b\right\rangle_{t}=0
$$

which applies to TMSV, and from the Heisenberg equations of motions of $a$ and $b$, the time-dependence of the second moments are given by:

$$
\begin{aligned}
\left\langle x^{2}\right\rangle_{t} & =\left\langle x^{2}\right\rangle_{0} \eta(t)+\frac{G+L}{2(G-L)}[\eta(t)-1], \\
\langle x y\rangle_{t} & =\langle x y\rangle_{0} \eta(t) .
\end{aligned}
$$

Here the initial second moments are given by:

$$
\begin{aligned}
\left\langle x^{2}\right\rangle_{0} & =\frac{2 A_{0}-C_{0}}{2\left[\left(2 A_{0}-C_{0}\right)^{2}-\left(B_{0}+D_{0}\right)^{2}\right]}, \\
\langle x y\rangle_{0} & =\frac{B_{0}+D_{0}}{2\left[\left(2 A_{0}-C_{0}\right)^{2}-\left(B_{0}+D_{0}\right)^{2}\right]} .
\end{aligned}
$$

Thus the time evolution of the coefficients of the twomode Gaussian state solution are as follows:

$$
\begin{aligned}
A(t)= & \frac{1}{4}\left[\left(2 A_{0}+C_{0}\right) \eta+\frac{G+L}{G-L}(\eta-1)\right. \\
& \left.+\frac{\left\langle x^{2}\right\rangle_{t}}{2\left(\left\langle x^{2}\right\rangle_{t}^{2}-\langle x y\rangle_{t}^{2}\right)}\right], \\
B(t)= & \frac{1}{2}\left[\left(B_{0}-D_{0}\right) \eta+\frac{\langle x y\rangle_{t}}{2\left(\left\langle x^{2}\right\rangle_{t}^{2}-\langle x y\rangle_{t}^{2}\right)}\right], \\
C(t)= & \frac{1}{2}\left[\left(2 A_{0}+C_{0}\right) \eta+\frac{G+L}{G-L}(\eta-1)\right. \\
& \left.-\frac{\left\langle x^{2}\right\rangle_{t}}{2\left(\left\langle x^{2}\right\rangle_{t}^{2}-\langle x y\rangle_{t}^{2}\right)}\right], \\
D(t)= & \frac{1}{2}\left[-\left(B_{0}-D_{0}\right) \eta+\frac{\langle x y\rangle_{t}}{2\left(\left\langle x^{2}\right\rangle_{t}^{2}-\langle x y\rangle_{t}^{2}\right)}\right],
\end{aligned}
$$

where the expectation values are given in Eq. (A5).

In the case of TMSV, the solution is reduced to Eq. (12) as the initial coefficients satisfy:

$$
\begin{aligned}
& C_{0}=D_{0}=0 \\
& 4 A_{0}^{2}-B_{0}^{2}=1 .
\end{aligned}
$$

In particular, by noting that $A_{0}=\left\langle x^{2}\right\rangle_{0}=\frac{1}{2}+\left\langle a^{\dagger} a\right\rangle_{0}=$ $\frac{1}{2}+\frac{\lambda^{2}}{1-\lambda^{2}}$, we have $A_{0}=\frac{1}{2} \cosh 2 r$.

\section{APPENDIX B: DERIVATION OF EIGENVECTORS AND EIGENVALUES OF $\rho^{T_{A}}$}

In position space, $\rho^{T_{A}}\left(x_{1}, y_{1} ; x_{2}, y_{2} ; t\right)=$ $\rho\left(x_{2}, y_{1} ; x_{1}, y_{2} ; t\right)$. The eigenvectors $\varphi_{n m}$ and eigenvalues $\xi_{n m}$ of $\rho^{T_{A}}$ are defined by Eq. (13). Applying the transformation $x_{j}=\left(u_{j}+v_{j}\right) / \sqrt{2}$ and $y_{j}=\left(-u_{j}+v_{j}\right) / \sqrt{2}$ $(j=1,2), \rho^{T_{A}}$ becomes a neat product of two double Gaussians as follows:

$$
\begin{aligned}
& \rho^{T_{A}}\left(u_{1}, v_{1} ; u_{2}, v_{2} ; t\right) \\
& =\Xi(t) \exp \left[-\alpha_{1}(t)\left(u_{1}-u_{2}\right)^{2}-\beta_{1}(t)\left(u_{1}+u_{2}\right)^{2}\right] \\
& \times \exp \left[-\alpha_{2}(t)\left(v_{1}-v_{2}\right)^{2}-\beta_{2}(t)\left(v_{1}+v_{2}\right)^{2}\right]
\end{aligned}
$$

where $\alpha_{1}=\frac{1}{4}(2 A-B+C+D), \beta_{1}=\frac{1}{4}(2 A+B-C+D)$, $\alpha_{2}=\frac{1}{4}(2 A+B+C-D)$ and $\beta_{2}=\frac{1}{4}(2 A-B-C-D)$. 
We apply Mehler's Formula twice, one for the $u_{1}, u_{2}$ double Gaussian, and one for the $v_{1}, v_{2}$ double Gaussian function. This would lead to the Schmidt decomposition on $\rho^{T_{A}}$ :

$$
\begin{aligned}
& \rho^{T_{A}}\left(u_{1}, v_{1} ; u_{2}, v_{2} ; t\right)=\Xi(t) \frac{\pi}{4}\left(\frac{1}{\alpha_{1} \beta_{1} \alpha_{2} \beta_{2}}\right)^{\frac{1}{4}} \\
& \times \sum_{n=0}^{\infty} \lambda_{n} f_{n}\left(u_{1}\right) f_{n}\left(u_{2}\right) \sum_{m=0}^{\infty} \tilde{\lambda}_{m} \tilde{f}_{m}\left(v_{1}\right) \tilde{f}_{m}\left(v_{2}\right),
\end{aligned}
$$

where the Schmidt modes $f_{n}(u)$ and $\tilde{f}_{m}(u)$ are:

$$
\begin{aligned}
f_{n}(u)= & \frac{1}{\sqrt{2^{n-1} n !}}\left(\frac{\sqrt{\alpha_{1} \beta_{1}}}{\pi}\right)^{\frac{1}{4}} H_{n}\left[2\left(\alpha_{1} \beta_{1}\right)^{\frac{1}{4}} u\right] \\
& \exp \left(-2 \sqrt{\alpha_{1} \beta_{1}} u^{2}\right) \\
\tilde{f}_{m}(u)= & \frac{1}{\sqrt{2^{m-1} m !}}\left(\frac{\sqrt{\alpha_{2} \beta_{2}}}{\pi}\right)^{\frac{1}{4}} H_{m}\left[2\left(\alpha_{2} \beta_{2}\right)^{\frac{1}{4}} u\right] \\
& \exp \left(-2 \sqrt{\alpha_{2} \beta_{2}} u^{2}\right)
\end{aligned}
$$

and the coefficients $\lambda_{n}(t)$ and $\tilde{\lambda}_{m}(t)$

$$
\begin{aligned}
& \lambda_{n}(t)=2\left(\alpha_{1} \beta_{1}\right)^{\frac{1}{4}} \frac{\left(\sqrt{\alpha_{1}}-\sqrt{\beta_{1}}\right)^{n}}{\left(\sqrt{\alpha_{1}}+\sqrt{\beta_{1}}\right)^{n+1}}, \\
& \tilde{\lambda}_{m}(t)=2\left(\alpha_{2} \beta_{2}\right)^{\frac{1}{4}} \frac{\left(\sqrt{\alpha_{2}}-\sqrt{\beta_{2}}\right)^{m}}{\left(\sqrt{\alpha_{2}}+\sqrt{\beta_{2}}\right)^{m+1}},
\end{aligned}
$$

where $H_{n}(u)$ are the Hermite polynomials. Rearranging terms, Eq. (B2) gives:

$$
\begin{aligned}
& \rho^{T_{A}}\left(x_{1}, y_{1} ; x_{2}, y_{2} ; t\right) \equiv \sum_{n, m} \xi_{n, m}(t) \varphi_{n, m}\left(x_{1}, y_{1} ; t\right) \\
&\left.\times \varphi_{n, m}\left(x_{2}, y_{2} ; t\right), \quad \text { (B } 5\right)
\end{aligned}
$$

where the eigenvectors $\varphi_{n, m}$ are

$$
\begin{aligned}
& \varphi_{n, m}\left(x_{1}, y_{1} ; t\right) \\
& =\frac{1}{\sqrt{2^{n-1} 2^{m-1} n ! m !}}\left(\frac{\sqrt{\alpha_{1} \beta_{1} \alpha_{2} \beta_{2}}}{\pi^{2}}\right)^{\frac{1}{4}} \\
& \times H_{n}\left[2\left(\alpha_{1} \beta_{1}\right)^{\frac{1}{4}}\left(\frac{x_{1}-y_{1}}{\sqrt{2}}\right)\right] H_{m}\left[2\left(\alpha_{2} \beta_{2}\right)^{\frac{1}{4}}\left(\frac{x_{1}+y_{1}}{\sqrt{2}}\right)\right] \\
& \times \exp \left[-\sqrt{\alpha_{1} \beta_{1}}\left(x_{1}-y_{1}\right)^{2}\right] \exp \left[-\sqrt{\alpha_{2} \beta_{2}}\left(x_{1}+y_{1}\right)^{2}\right],
\end{aligned}
$$

and the eigenvalues $\xi_{n, m}$ of $\rho^{T_{A}}$ are,

$$
\xi_{n, m}(t)=\Xi(t) \pi \frac{\left(\sqrt{\alpha_{1}}-\sqrt{\beta_{1}}\right)^{n}}{\left(\sqrt{\alpha_{1}}+\sqrt{\beta_{1}}\right)^{n+1}} \frac{\left(\sqrt{\alpha_{2}}-\sqrt{\beta_{2}}\right)^{m}}{\left(\sqrt{\alpha_{2}}+\sqrt{\beta_{2}}\right)^{m+1}} .
$$

These expressions of eigenvectors and eigenvalues are for any time-dependent coefficients $A(t), B(t), C(t)$ and $D(t)$, i.e., applicable to states evolving from arbitrary initial values $A_{0}, B_{0}, C_{0}$ and $D_{0}$. The special case with initial TMSV is given in Eq. (14) and Eq. (18).
[1] S. L. Braunstein, and P. van Loock, Rev. Mod. Phys. 77, 513 (2005).

[2] A. Furusawa, J. L. Sørensen, S. L. Braunstein, C. A. Fuchs, H. J Kimble, and E. S. Polzik, Science 282, 706 (1998).

[3] W. P. Bowen, N. Treps, B. C. Buchler, R. Schnabel, T. C. Ralph, Hans-A. Bachor, T. Symul, and P. K. Lam, Phys. Rev. A 67, 032302 (2003).

[4] T. C. Zhang, K. W. Goh, C. W. Chou, P. Lodahl, and H. J. Kimble, Phys. Rev. A 67, 033802 (2003).

[5] Nobuyuki Takei, Hidehiro Yonezawa, Takao Aoki, and Akira Furusawa, Phys. Rev. Lett. 94, 220502 (2005).

[6] Hidehiro Yonezawa, Takao Aoki, and Akira Furusawa, Nature 431, 430 (2004).

[7] X. Li, Q. Pan, J. Jing, J. Zhang, C. Xie, and K. Peng, Phys. Rev. Lett. 88, 047904 (2002).

[8] J. Mizuno, K. Wakui, A. Furusawa, and M. Sasaki, Phys. Rev. A 71, 012304 (2005).

[9] G. Giedke, M. M. Wolf, O. Kruger, R. F. Werner, and J. I. Cirac, Phys. Rev. Lett. 91, 107901 (2003).

[10] T. Hiroshima, Phys. Rev. A 63, 022305 (2001).
[11] A. K. Rajagopal and R. W. Rendell, Phys. Rev. A 63, 022116 (2001).

[12] P. J. Dodd and J. J. Halliwell, Phys. Rev. A 69, 052105 (2004); P. J. Dodd, Phys. Rev. A 69, 052106 (2004).

[13] S. Scheel and D.-G. Welsch Phys. Rev. A 64, 063811 (2001).

[14] S. J. van Enk, and O. Hirota, Phys. Rev. A 71, 062322 (2005).

[15] A. Serafini, F. Illuminati, M. G. A. Paris, and S. De Siena, Phys. Rev. A 69, 022318 (2004); A. Serafini, M. G. A. Paris, F. Illuminati, and S. De Siena, J. Opt. B: Quantum Semiclass. Opt. 7, R19 (2005).

[16] M. Ban, J. Phys. B 39, 1125 (2006).

[17] J. S. Prauzner-Bechcicki, J. Phys. A 37, L173 (2004).

[18] S. Daffer, K. Wódkiewicz, and J. K. McIver, Phys. Rev. A 68, 012104 (2003).

[19] Xiao-Yu Chen, J. Phys. B 39, 4605 (2006); Xiao-Yu Chen, Phys. Rev. A. 73, 022307 (2006).

[20] A. Peres, Phys. Rev. Lett. 77, 1413 (1996).

[21] M. Horodecki, P. Horodecki, and R. Horodecki, Phys. Lett. A 223, 1 (1996). 
[22] P. Horodecki, Phys. Lett. A 232, 333 (1997).

[23] G. S. Agarwal and A. Biswas, New J. Phys. 7, 211 (2005).

[24] E. Shchukin and W. Vogel, Phys. Rev. Lett. 95, 230502 (2005).

[25] R. Simon, Phys. Rev. Lett. 84, 2726 (2000).

[26] L. M. Duan, G. Giedke, J. I. Cirac, and P. Zoller, Phys. Rev. Lett. 84, 2722 (2000).

[27] For a review, see A. Ferraro, S. Olivares, and M. G. A. Paris, Gaussian States in Quantum Information, Napoli Series on physics and Astrophysics, (Bibliopolis, Napoli, 2005) and references therein.

[28] G. Vidal and R. F. Werner, Phys. Rev. A 65, 032314 (2002).

[29] V. Vedral and M. B. Plenio, Phys. Rev. A 57, 1619 (1998).

[30] X. B. Wang, M. Keiji, and T. Akihisa, Phys. Rev. Lett. 87, 137903 (2001).

[31] See for example, O. Gühne, P. Hyllus, D. Bruss, A. Ekert, M. Lewenstein, C. Macchiavello, and A. Sanpera, Phys. Rev. A 66, 062305 (2002).
[32] Z. B. Chen, J. W. Pan, G. Hou, and Y. D. Zhang, Phys. Rev. Lett. 88, 040406 (2002).

[33] L. Mandel and E. Wolf, Optical Coherence and Quantum Optics, (Cambridge University Press, New York, 1995).

[34] Stein Olav Skrøvseth, Phys. Rev. A 72, 062305 (2005).

[35] Let $|\phi\rangle \equiv \sum_{n m} c_{n m}|n\rangle|m\rangle$ be an eigenvector of $\rho^{T_{A}}$ obtained from the partial transposition of $\rho$ in Fock space, then it can be shown that $|\tilde{\phi}\rangle=$ $\sum_{n m} c_{n m} \sum_{k}\langle n \mid \tilde{k}\rangle|\tilde{k}\rangle \sum_{j}\langle\tilde{j} \mid m\rangle|\tilde{j}\rangle$ is the corresponding eigenvector of $\rho^{T_{A}}$ obtained from the partial transposition of $\rho$ in the new bases (labeled by tilde), with the same eigenvalue. Therefore $|\phi\rangle$ and $|\tilde{\phi}\rangle$ are related by a direct bases transformation if $\langle n \mid \tilde{k}\rangle=\langle\tilde{k} \mid n\rangle$, i.e., when $\langle n \mid \tilde{k}\rangle$ is real.

[36] K. Życzkowski, P. Horodecki, A. Sanpera, and M. Lewenstein, Phys. Rev. A 58, 883 (1998).

[37] G. M. DAriano, C. Macchiavello, and M. G. A. Paris, Phys. Rev. A 67, 042310 (2003). 